\title{
Why Deep Space Habitats Should be Different from the International Space Station
}

\author{
Brand Griffin \\ MacAulay Brown \\ Jacobs ESSSA Group
}

It is tempting to view the International Space Station (ISS) as a model for deep space habitats. This is not a good idea for many reasons. The ISS does not have a habitation module; instead the individual crew quarters are dispersed across several modules, the galley is in the US Laboratory and the waste hygiene compartment is in a Node. This distributed arrangement may be inconvenient but more important differences distinguish a deep space habitat from the ISS. First, the Space Shuttle launch system that shaped, sized, and delivered most ISS elements has been retired. Its replacement, the Space Launch System (SLS), is specifically designed for human exploration beyond low-Earth orbit and is capable of transporting more efficient, large diameter, heavy-lift payloads. Next, because of the Earth's protective geomagnetic field, ISS crews are naturally shielded from lethal radiation. Deep space habitat designs must include either a storm shelter or strategically positioned equipment and stowage for radiation protection. Another important difference is the increased transit time with no opportunity for an ISS-type emergency return. It takes 7 to 10 days to go between Earth and cis-lunar locations and 1000 days for the Mars habitat transit. This long commute calls for greater crew autonomy with habitats designed for the crew to fix their own problems. The ISS rack-enclosed, densely packaged subsystems are a product of the Shuttle era and not maintenance friendly. A solution better suited for deep space habitats spreads systems out allowing direct access to single-layer packaging and providing crew access to each component without having to remove another. Operational readiness is another important discriminator. The ISS required over 100 flights to build, resupply, and transport the crew, whereas SLS offers the capability to launch a fully provisioned habitat that is operational without additional outfitting or resupply flights. 